\title{
The Role of IL-21 in Chronic Inflammatory Skin Diseases
}

\author{
Elisabetta Botti • Andreea N. Boca • Giulia Spallone • \\ Giovanni Monteleone • Antonio Costanzo
}

Published online: 15 December 2012

(C) Springer Science+Business Media New York 2012

\begin{abstract}
Interleukin (IL)-21 is a type I cytokine involved in both innate and adaptive immunity. In structure, due to the common $\gamma$-chain, it shares significant homology with IL-2, IL-4, and IL-15 but binds to a unique receptor chain, IL-21R, activating the Jak/STAT pathway. Like most cytokines, IL-21 exhibits a plethora of actions, modulating both humoral and cell-mediated immune responses. Summing, it has effect on lineages, such as CD4 and CD8 T cells, regulatory $\mathrm{T}$ cells, $\mathrm{B}$ cells, natural killer cells, and dendritic cells, as well as on nonimmune cells. The variety of effects that IL-21 has on the immunity explains its critical role in the initiation and progression of several inflammatory and autoimmune diseases. Several studies support its involvement in the pathogenesis of inflammatory bowel disease, celiac disease, rheumatoid arthritis, type I diabetes, and some inflammatory skin disorders, even if the exact mechanism is not fully understood. We will summarize recent discoveries on the role of IL-21 in inflammatory skin diseases.
\end{abstract}

Keywords Psoriasis · IL-21 · Inflammation · Skin · STAT3 · SLE $\cdot$ Urticaria $\cdot$ Atopic dermatitis

\footnotetext{
E. Botti $\cdot$ G. Spallone $\cdot$ A. Costanzo $(\bowtie)$

Department of Dermatology, University of Rome

"Tor Vergata", Viale Oxford 81,

00133 Rome, Italy

e-mail: antonio.costanzo@uniroma2.it

A. N. Boca

Department of Pharmacology, Toxicology and Clinical

Pharmacology, University of Medicine and Pharmacy

"Iuliu Hatieganu", Cluj-Napoca, Romania

G. Monteleone

Department of Gastroenterology, University of Rome

"Tor Vergata", Rome, Italy
}

\section{Introduction}

Interleukin (IL)-21 is a cytokine with pleiotropic effects on several immune and nonimmune cells and whose production is deregulated in many pathological conditions [1]. Chronic inflammatory skin diseases are a broad group that share similar characteristics with other inflammatory/autoimmune disorders, including a complex pathogenesis often with unknown etiology, systemic inflammation, and multiorgan involvement. In recent years, new insights into the comprehension of IL-21 functions have advanced our understanding of the pathology of these skin diseases (Fig. 1).

\section{Overview of IL-21 Structure and Functions}

The IL-21 gene is located on human chromosome 4q26-q27 and encodes a protein of 162 amino acids. IL-21 is a member of the $\gamma$-chain cytokine family, which also includes IL-2, IL-4, IL-7, IL9, IL-13, and IL-15. IL-21 shares sequence homology with IL-2, IL-4, and IL-15. It binds to and forms a complex with the IL-21 receptor (IL-21R), which is composed of two subunits, a unique IL-21 $\alpha$ chain (IL-21R) and the common cytokine receptor $\gamma$-chain $(\gamma \mathrm{c})$ [2]. Binding of IL-21 to the IL-21R $\alpha / \gamma$ complex activates Janus tyrosine kinase 3 (Jak3) and Jak1. Subsequently, Jak1 and Jak3 themselves activate signal transducer and activator of transcription 1 (STAT1) and STAT3 respectively and, to a lesser extent, STAT4 and STAT5 [3, 4]. On the contrary other $\gamma$-chain cytokines activate predominantly STAT5, and this could explain the different effects of these cytokines on immune cells [5]. Like the majority of cytokines, IL-21 exhibits a wide range of actions depending on environmental signals and may modulate both humoral and cellmediated immune responses. IL-21 has effect on multiple lineages, including $\mathrm{CD} 4$ and $\mathrm{CD} 8 \mathrm{~T}$ cells, regulatory $\mathrm{T}$ 


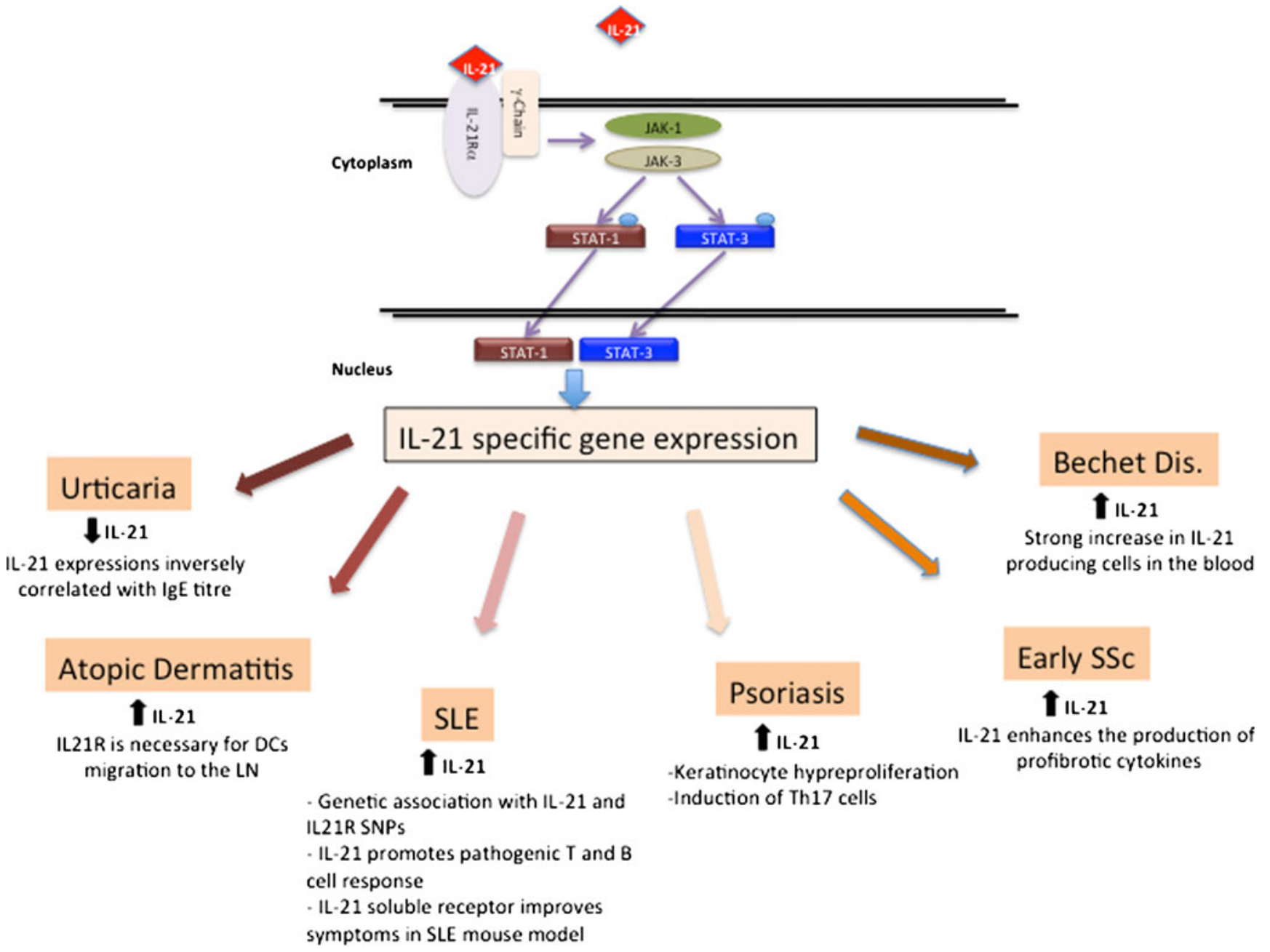

Fig. 1 Schematic diagram showing IL-21 activated signaling pathway and key findings on IL-21 role in the pathogenesis of chronic inflammatory skin diseases

(Tregs) cells, B cells, natural killer (NK) cells and dendritic cells (DC), as well as on nonimmune cells, such as epithelial cells, fibroblast-like synovial cells, stromal cells, and endothelial cells [6]. IL-21 has been involved with transforming growth factor-beta (TGF- $\beta$ ) in differentiation of human naïve CD4 T cells into Th-17 cells [7-9]. Moreover, IL-21 induces its own expression via an autocrine loop and upregulates the IL-23R expression; IL-23 is known to be essential for the amplification of Th17 cell programs. Indeed, IL21-deficient cells fail to express IL23R [8]. IL-21 expression and Th17 differentiation require the presence of transcription factor interferon regulatory factor 4 (IRF4), which regulates the expression of ROR $\gamma \mathrm{t}$ and ROR $\alpha$ and directly binds to the IL-21 promoters $[10,11]$. Studies in human cells have shown that IL-21 also can promote Th1 differentiation, enhance the expression of Th1-associated transcription factors, such (i.e., STAT4 and T-bet) and increase interferon- $\gamma($ IFN- $\gamma)$ production $[12,13]$. IL-21 also may negatively regulate the maturation and function of DC $[14,15]$. Additionally, IL-21 antagonizes TGF- $\beta$-mediated
Foxp3 expression, thus inhibiting peripheral differentiation of regulatory $\mathrm{T}$ cells (Tregs) [16]. This activity seems to be mediated by IRF4, as IL-21 fails to suppress Foxp3 in IRF4deficient $T$ cells [17]. On the contrary, other data suggest that IL-21 may actually promote Tregs' function [18]. Some data indicate that IL-21 contributes to the generation of IL10-producing Tregs type 1 ( $\operatorname{Tr} 1)$, which display a suppressive properties on effector T cells [19•]. This is consistent with a study showing that IL-21 stimulates IL-10 production, a central cytokine involved in immunosuppressive actions [20•]. On the other hand, it has been revealed that IL-21 makes CD4 T cells resistant to the suppressive effects of Tregs without directly reversing the function of these cells [21]. Furthermore, IL-21 plays a crucial role in regulating B cells proliferation; it also is required for normal germinal center (GC) formation, plasma cell differentiation, immunoglobulin production, and isotype switching toward $\operatorname{IgG}$, IgM, and IgA [22-24]. IL-21 is produced by Th17, Th1, and NK T cells $[7,8,13,16,25]$. Recently, it has been shown that it is highly produced by $\mathrm{T}$ follicular helper ( $\mathrm{Tfh}$ ) 
cells, which are a distinct subset of CD4 T cells that express CXC chemokine receptor 5 (CXCR5) and inducible T cell costimulator (ICOS). In the lymphoid follicles, Tfh cells promote $\mathrm{B}$ cells proliferation and cell-dependent antibody response [26-28, 29••]. IL21R knockout mice display normal B cell subset but abnormal B cell function, with an elevated production of IgE and diminished IgG levels [30].

Given the variety of effects that IL-21 has on the immune system, it is not surprising that this cytokine has a critical role in the initiation and progression of inflammatory reactions involved in several immune-mediated diseases. Indeed, several studies on the genetic association or murine models have suggested a role of IL-21 in the pathogenesis of inflammatory bowel disease, celiac disease, rheumatoid arthritis, type I diabetes, and some inflammatory skin disorders [31-35], even if the mechanisms involved in IL-21 actions are multiple and often conflict with each other.

\section{IL-21 in Chronic Inflammatory Skin Diseases}

\section{Psoriasis}

Psoriasis is a polygenic, multifactorial, immune-mediated disorder and may be considered as a prototype of chronic inflammatory skin diseases. During the development of the psoriasis lesions, environmental triggers operate on a complex genetic background with initial activation of innate immune response mediated by DC, which subsequently migrate into the lymph nodes, where they induce naïve Tcells differentiation toward Th1 and Th17 effector cells. The engagement of adaptive immune system leads to the production of specific inflammatory cytokines and to keratinocyte proliferation, establishing a vicious circle with an autoamplification loop. In this context, a variety of cytokines play a crucial role in the modulation of gene expression in the target cells [36]. We have shown that IL-21 is highly expressed in skin and peripheral blood of psoriatic patients compared with nonlesional skin and healthy controls. This cytokine was mostly produced by CD4 T cells, which also are increased in psoriatic patients and to a lesser extent by NKT cells. Moreover, keratinocytes expressed IL-21R and underwent hyperproliferation when stimulated with IL-21. In vivo, IL-2 1 causes psoriasis-like hyperplasia when injected intradermally into mice and this is accompanied by increased levels of inflammatory cytokines, such as IFN- $\gamma$, IL-17A, and IL-22. Furthermore, in the human psoriasis-xenograft mouse model, antibody-mediated blockade of IL-21 activity leads to a resolution of inflammation with reduced level of Th1 and Th17 cytokines and also results in decreasing of keratinocyte proliferation [37•]. In a subsequent study, we provided evidence that skin CD4 recruitment is required for IL-21-induced hyperplasia in mice. CD4 recruitment is mediated by chemokine $\mathrm{C}-\mathrm{C}$ motif ligand 27 (CCL27) expression, whereas epidermal changes are mostly due to INF- $\gamma$ production [38]. These results taken collectively support an important role for IL-21 in the pathogenesis of psoriasis and suggest that blockade of IL-21 may be therapeutically effective in psoriatic patients. In addition, in psoriasis IL-21 also may act by influencing different pathways. In fact, as mentioned above, it may negatively regulate the differentiation of Tregs and also make $\mathrm{T}$ cells resistant to the suppressive effects of these Tregs [21]; these biological phenomenon have been documented in psoriasis [39]. Moreover, IL-21 could play a role in the differentiation of Th17 cells, which have implicated in development of psoriasis [40].

\section{Systemic Lupus Erythematous}

Systemic lupus erythematous (SLE) is a chronic multiorgan inflammatory disease of unknown origin that affects most commonly skin, joints, kidneys, hematologic system, lungs, and central nervous system. SLE is characterized by deregulated interaction between T cells and B cells with antinuclear antibody $(\mathrm{Ab})$ production and immune-complex deposition leading to tissue damage. A variety of different cytokines are crucial in this pathological T and B cells cross-talking. Among these inflammatory cytokines, IL-21 seems to play a relevant role in the pathogenesis of SLE. Patients affected by SLE display an increased serum level of IL-21 compared with healthy controls, but IL-21 levels do not seem to correlate with SLE activity [41]. According to these data, we also showed that IL-21 expression is enhanced in skin samples from patients affected by SLE [37•]. Notably, IL-21expressing CD4 $\mathrm{T}$ cells are increased in SLE patients, and there is a correlation between IL-21 CD4 T cells and IL-17A CD4 T cells in SLE, whereas no differences are seen in IL21R expression on CD19, CD4, and CD8 cells between SLE patients and healthy controls [42]. Genetic association of IL21 and IL-21R polymorphisms also has been demonstrated in SLE $[43,44]$. Remarkably, in some studies on SLE patients, an increased number of $\mathrm{Tfh}$ cells and decreased number of Tregs were found [45-47]. Moreover several works using various mouse models of SLE provided new insights into the role of IL-21 in the pathogenesis of this disorder.

MRL/MpJ-Fas lpr/lpr /J (MRL lpr) mice develop a spontaneous autoimmune disease characterized by activation of $\mathrm{B}$ and $\mathrm{T}$ cells, production of anti-ds-DNA antibodies, skin lesions, splenomegaly, lymphadenopathy, and glomerulonephritis, which with age increase significantly in severity and resemble human SLE [48•]. MRL lpr mice show increased number of follicular $\mathrm{T}(\mathrm{Tfh})$ cells and extrafollicular T cells; the latter express ICOS but do not express CXCR5. In this mouse model, ICOS and CD4 T cells are the primary source for IL-21 production and for subsequent extrafollicular 
plasmablast development. This extrafollicular population also is able to produce IL- 4 and INF- $\gamma$. The relationship between these extrafollicular $\mathrm{T}$ cells and the other $\mathrm{T}$ populations remains to be determined [49]. In a recent work using MRL lpr IL-21R knockout mice (MRL lpr IL-21R KO), it has been shown that SLE-like clinical features are decreased compared to wild-type mice. Furthermore, the number of splenocytes, total plasma cells, IgG1, IgG2a, IgG3, and IgM serum titers anti-nuclear and anti-dsDNA Abs from MRL lpr IL-21R KO mice are reduced and germinal center formation is absent. This suggests that IL-21R contributes to the development of splenomegaly and glomerulonephritis in this mouse model, regulating $\mathrm{B}$ cell activation and autoantibodies production. Indeed, INF- $\gamma$ producing CD4 T cells and IL-4 producing CD4 T cell are decreased in MRL lpr IL-21R KO mice. On the contrary, IL-17-producing CD4 $+\mathrm{T}$ cells are increased [30]. Taken together, these data indicate that IL-21 drives systemic autoimmunity in the MRL lpr model of SLE by promoting both pathogenic B- and T-cell responses in these mice. In other studies on MRL lpr mice, administration of IL$21 \mathrm{R} / \mathrm{Fc}$ fusion protein that blocks IL-21 signaling strongly reduces proteinuria, skin lesions, lymphadenopathy, circulating autoantibodies, and total IgG. Treating MRL lpr mice with IL-21R/Fc treatment also leads to a reduction of both splenic T-cell and B-cell antibodies production [48•]. Investigations in BXSB.B6-Yaa $+/ \mathrm{J}$ mice, which also develop a disorder very similar to human SLE [50] show an increased serum IL-21, IgG1, and IgG3 expression [24]. As in MRL lpr mice, IL-21 are produced by extrafollicular Tfh cells [51]. SLE-like symptoms are prevented in IL-21R-deficient BXSB-Yaa $+/ \mathrm{J}$ mice. Notably, treatment of these mice with a fusion protein blocking IL-21 leads to a decrease of IL-21, IgG1 production, and T-cells activation. It has to be underlined that block of IL- 21 in the early stage of disease negatively influences survival, whereas the administration in the later phase is favorable [52•]. This double role of IL-21 is not clear, but a possible explanation as described by Monteleone and collaborators could be that IL-21 may exert both inflammatory and antiinflammatory effects, and this seems to be related to the capability of IL-21 to stimulate IL-10 production, which is highly expressed in patients affected by SLE and in BXSB$\mathrm{Yaa}+/ \mathrm{J}$ mice $[20 \bullet, 53]$.

In conclusion, these studies suggest a critical role of IL21/IL-21R pathway in the pathogenesis of SLE, and although mouse model do not reflect the complexity of human disease, IL-21 blockade might be a promising approach to treat SLE, even if further investigation are necessary to confirm a possible therapeutic use.

\section{Atopic Dermatitis}

Atopic dermatitis (AD) is a chronic and recurrent inflammatory skin disease, characterized by pruritic eczematous skin lesions. The etiology and pathogenesis of the disease remain incompletely understood, but it is clear the role of genetic predisposition, impaired skin barrier function, and allergic response against allergens. It has been known that $\mathrm{AD}$ is related to increased production of $\mathrm{IgE}$ from B cells, and this production depends on cytokines produced by $\mathrm{T}$ cells, especially IL-4/IL-13 [54, 55]. Acute lesions seem to be associated to Th2 response, whereas chronic phase is associated to Th1 profile [56, 57]. Recent studies provide insights into immunological mechanisms, including various cytokines in skin and peripheral blood that lead to the atopic skin inflammation. Jin and collaborators noticed that IL-21 and IL-21R are up-regulated in acute skin lesions compared to healthy control skin [58•]. Our data [37•] also confirmed the observation of increased IL-21 expression in skin biopsies taken from AD patients. IL-21 is expressed by lymphocytes in the inflamed dermis, whereas IL-21R is expressed in epidermal keratinocytes and along the dermal-epidermal junction. Furthermore, IL-21 and IL-21R expression also is up-regulated in a mouse model of allergic skin inflammation elicited by application of Th2 ovalbumin (OVA) to tapestripped skin, which mimics many features of $\mathrm{AD}[59,60]$. It was previously reported that IL-21 is expressed by CD4 T cells after antigen receptor stimulation and also after IL-6 stimulation $[8,9]$. This study also indicates that tape stripping strongly upregulated IL-6 expression in the skin. In IL21R deficient mice OVA epicutaneous allergic sensitization is profoundly impaired, showing no eczematous changes and weak infiltration of CD4 T cells and eosinophils. Moreover, the expression of CCL17, a ligand for CCR4, which is expressed on skin homing $\mathrm{T}$ cells, is not increased and there is no detectable upregulation of IL-4, IL-13, or IFN- $\gamma$ mRNA. At the same time, IL-21R knockout mice do not develop OVA-specific IgG1 and IgG2a antibody responses. The authors conclude that the absence of IL-21R impairs the development of a systemic response to epicutaneous allergic sensitization. Furthermore, they suggest that IL-21R plays an important role in the migration of antigen-presenting DCs from skin to draining lymph nodes (DLNs), but not in their expression of activation markers. In IL-21R-deficient mice, DC in DLNs do not express CCR7 and there is no activation of matrix metalloproteinase 2 (MMP2) compared with wildtype mice, plus both CCR7 and MMP2 are required for the migration of DCs from skin to LN. To confirm the role of IL-21 in the pathogenesis of AD, soluble IL-21R-IgG2aFc fusion protein, which blocks IL-21/IL-21R interaction, was used in OVA epicutaneous allergic sensitization mouse model. It indeed inhibited allergic skin inflammation, decreasing infiltration of CD4 T cells and eosinophils' expression of Th2-related cytokines, IL-4, and IL-13. Moreover, treatment with soluble IL-21R-IgG2aFc fusion protein decreased $\operatorname{IgG} 1$ and $\operatorname{IgG} 2$, but not $\operatorname{IgE}$, and also inhibits DC trafficking. Splenocytes from IL21R deficient mice fail to 
proliferate and secrete both Th2 and Th1 cytokines in response to OVA stimulation in vitro [58•]. There are conflicting data on the effects of IL-21 on allergic skin inflammation. It has been reported that IL-21-treated DCs fail to induce hapten-induced contact hypersensitivity reactions via inhibition of DC activation and maturation in vivo [14]. Another study shows a decreased level of IL-21 in peripheral blood of patients with AD compared with normal control. It is worth mentioning that the level of IL-21 is inversely correlated to the severity of skin disease. These studies suggest a role of IL21 in the pathogenesis of AD with a probable high level in skin lesions but low level in peripheral blood [61].

\section{Urticaria}

Urticaria is characterized by transient inflammation with swellings of the skin or mucosa, mouth, and genitalia, due to a transient leakage of plasma from small blood vessels into the surrounding connective tissues. It can occur acutely or evolve in a chronic mode. Urticaria results from immune and nonimmune activation of cutaneous mast cells and the release of inflammatory mediators, such as cytokines and eicosanoids. In a recent study, it has been demonstrated that T-cell activation is proportional to mast-cell deregulation in patients with chronic urticaria (CU), and T cells infiltrating the skin can participate in the chronicity of the lesions [62]. Another work from the same group has shown that serum level of IL-15 and IL-21 are significantly decreased in acute urticarial (AU) and $\mathrm{CU}$, whereas IgE production is increased. Moreover, a significant correlation is found between IL-21 and IgE in CU [63]. This may be explained by the lack of IL-21 activity promoting isotype switching and Th1 and Th17 differentiation, by this leading to a hyperfunction of the Th2 subtype [64].

\section{Systemic Sclerosis}

Systemic sclerosis ( $\mathrm{SSc}$ ) is an autoimmune connective tissue disease that affects the skin, blood vessels, and internal organs. The pathogenesis is still not fully elucidated. SSc is characterized by microvascular dysfunction, immune activation, and antibody production, which leads to collagen deposition and fibrosis [65]. It has been published that IL21R is highly expressed in the lesional epidermis, fibroblasts, and endothelial cells of SSc patients. The same expression pattern was seen in clinically uninvolved skin. On the contrary, IL-21 was not detected in the same samples. Moreover, in vitro treatment of keratinocytes with IL21 enhances the expression of the adhesion molecules, $\alpha-3$ integrin and $\alpha-6$ integrin, and vascular endothelial growth factor (VEGF), which are involved in angiogenesis and leukocyte migration. These results suggest that IL-21 may be involved in the pathogenesis of SSc via induction of VEGF. Notably, because IL-21 expression was not observed, it is not clear whether this cytokine is produced only in a specific phase or if IL-21R may bind different ligands other than IL-21 [66]. In addition, administration of recombinant IL-21 to culture media of SSc fibroblasts increased the production of IL-6, TGF-beta, and procollagen type I, and these seem to be augmented by prestimulation with endothelin-1 [67]. It has been postulated that in the early phases of SSc there is a predominance of Th1 and Th17 effectors, whereas in the later stages Th2 effectors are predominant [68]. IL17 and IL-23R are highly expressed in early stage of SSc, plus IL-17 may enhance the secretion of proinflammatory and profibrotic cytokines IL-6 and IL8 from fibroblasts [69]. As mentioned above, IL-21 contributes to Th17 differentiation and to IL-23R induction [7-9].

\section{Behçet's Disease}

Behçet's disease (BD) is a recurrent inflammatory multisystem disease of unknown etiology, characterized by oral and genital ulceration, ocular abnormalities, and cutaneous lesions. The pathogenesis involves vascular injuries and autoimmune response [70]. Cytotoxic CD8 T cells, Th1 cells, Th17 cells, and neutrophils appear to be responsible for the inflammatory lesions [71•]. High levels of IL-17 and IL-23 were recently reported in the peripheral blood from active stage of BD patients [72, 73]. Th17 responses and the suppression of Tregs correlate with BD activity and this may be mediated by IL-21. Notably, IL21 producing T cells are dramatically increased in peripheral blood of BD patients and correlate positively with Th17 and negatively with Tregs [74].

\section{Conclusions}

Several studies have provided evidence that IL-21 plays an essential role in a variety of chronic inflammatory skin diseases, acting on both immune and non-immune cells. Even if the complete understanding of these disorders remains still a challenge, the use of compounds neutralizing IL-21 (e.g., blocking antibodies, recombinant proteins) in some murine models of skin disease has underlined a promising advantage in the therapeutic use. However, because IL-21 is implicated in host defence against infection and tumors, further investigations are required to test the efficacy and potential adverse events in the clinical use.

Disclosures E. Botti, A.N. Boca, G. Spallone, and G. Monteleone reported no potential conflicts of interest relevant to this article. A. Costanzo has reported grants paid to his institution from the National 
Psoriasis Foundation, Italian Association for Cancer Research, and Telethon; he is a Young Dermatologist Board Member for Pfizer, and has received lecture payments from Janssen.

\section{References}

Papers of particular interest, published recently, have been highlighted as:

- Of importance

- Of major importance

1. Parrish-Novak J, Dillon S, Nelson A, et al. Interleukin 21 and its receptor are involved in $\mathrm{NK}$ cell expansion and regulation of lymphocyte function. Nature. 2000;408:57-63.

2. Ozaki K, Kikly K, Michalovich D, et al. Cloning of a type I cytokine receptor most related to the IL-2 receptor $\beta$ chain. Proc Natl Acad Sci U S A. 2000;97:11439-43.

3. Zeng R, Spolski R, Casas E, et al. The molecular basis of IL-21mediated proliferation. Blood. 2007;109:4135-42.

4. Wei L, Laurence A, Elias KM, et al. IL-21 is produced by Th17 cells and drives IL-17 production in a STAT3-dependent manner. J Biol Chem. 2007;282:34605-346010.

5. Gagnon J, Ramanathan S, Leblanc C, et al. Regulation of IL21 signaling by suppressor of cytokine signaling-1 (SOCS1) in CD8(+) T lymphocytes. Cell Signal. 2007;19:806-16.

6. Monteleone G, Pallone F, Macdonalds TT. Interleukin-21 as a new therapeutic target for immune-mediated disease. Trends Pharmacol Sci. 2009;30:441-7.

7. Yang L, Anderson DE, Baecher-Allan C, et al. IL-21 and TGF-beta are required for differentiation of human $\mathrm{T}(\mathrm{H}) 17$ cells.

8. Zhou L, Ivanov II, Spolski R, et al. IL-6 programs T(H)-17 cell differentiation by promoting sequential engagement of the IL-21 and IL-23 pathways. Nat Immunol. 2007;8:967-74.

9. Nurieva R, Yang XO, Martinez G, et al. Essential autocrine regulation by IL-21 in the generation of inflammatory T cells. Nature. 2007:448:480-3.

10. Brustle A, Heink S, Huber M, et al. The development of inflammatory $\mathrm{T}(\mathrm{H})-17$ cells requires interferon-regulatory factor 4 . Nat Immunol. 2007;8:958-66.

11. Chen Q, Yang W, Gupta S, et al. IRF-4 binding protein inhibits interleukin-17 and interleukin-21 production by controlling the activity of IRF-4 transcription factor. Immunity. 2008;299:899-911.

12. Strengell M, Matikainen S, Sirén J, et al. IL-21 in synergy with IL15 or IL-18 enhances IFN-g production in human NK and T cells. $J$ Immunol. 2003;170:5464-9.

13. Monteleone G, Monteleone I, Fina D, et al. Interleukin-21 enhances T-helper cell type I signaling and interferon-gamma production in Crohn's disease. Gastroenterology. 2005;128:687-94

14. Brandt K, Bulfone-Paus S, Foster DC, et al. Interleukin-21 inhibits dendritic cell activation and maturation. Blood. 2003;102:4090-8.

15. Strengell M, Lehtonen A, Matikainen S, et al. IL-21 enhances SOCS gene expression and inhibits LPS-induced cytokine production in human monocyte-derived dendritic cells. J Leukoc Biol. 2006;79:1279-85.

16. Korn T, Bettelli E, Gao W, et al. IL-21 initiates an alternative pathway to induce proinflammatory T(H)17 cells. Nature. 2007;448:484-7.

17. Huber M, Brüstle A, Reinhard K, et al. IRF4 is essential for IL-21mediated induction, amplification, and stabilization of the Th17 phenotype. Proc Natl Acad Sci U S A. 2008;105(52):20846-51.

18. Piao WH, Jee YH, Liu RL, et al. IL-21 modulates CD4+CD25+ regulatory $\mathrm{T}$-cell homeostasis in experimental autoimmune encephalomyelitis. Scand J Immunol. 2008;67:37-46.
19. - Pot C, Jin H, Awasthi A, et al. IL-27 induces the transcription factor c-Maf, cytokine IL-21 and costimulatory receptor ICOS that coordinately act together to promote differentiation of IL-10producing Tr1 cells. J Immunol. 2009;183(2):797-801. An interesting study that points out an anti-inflammatory actions of IL-21.

20. • Spolski R, Kim HP, Zhu W, et al. IL-21 mediates suppressive effects via its induction of IL-10. J Immunol. 2009;182:2859-67. An interesting study that points out an anti-inflammatory actions of IL-21.

21. Peluso I, Fantini MC, Fina D, et al. IL-21 counteracts the regulatory $\mathrm{T}$ cell mediated suppression of human CD4+ T lymphocytes. J Immunol. 2007;178:732-9.

22. Ettinger R, Kuchen S, Lipsky PE. The role of IL21 in regulating Bcell function in health and disease. Immunol Rev. 2008;223:60-86.

23. Konforte D, Simard N, Paige CJ. IL-21: an executor of B cell fate. J Immunol. 2009;182:1781-7.

24. Ozaki K, Spolski R, Ettinger R, et al. Regulation of B cell differentiation and plasma cell generation by IL-21, a novel inducer of Blimp-1 and Bcl-6. J Immunol. 2004;173:5361-71.

25. Coquet JM, Kyparissoudis K, Pellicci DG, et al. IL-21 is produced by NKT cells and modulates NKT cell activation and cytokine production. J Immunol. 2007;178:2827-34.

26. Bryant VL, Ma CS, Avery DT, et al. Cytokine-mediated regulation of human B cell differentiation into Ig-secreting cells: predominant role of IL-21 produced by CXCR5 T follicular helper cells. J Immunol. 2007;179:8180-90.

27. Nurieva RI, Chung Y, Hwang D, et al. Generation of T follicular helper cells is mediated by interleukin-21 but independent of T helper 1, 2, or 17 cell lineages. Immunity. 2008;29:138-43.

28. Vogelzang A, McGuire HM, Yu D, et al. A fundamental role for interleukin 21 in the generation of $\mathrm{T}$ follicular helper cells. Immunity. 2008;29:127-33.

29. •- Spolski R, Leonard WJ. IL-21 and T follicular helper cells. Int Immunol. 2010;22:7-12. A review that gives a very good overview on $I L-21$ and $B$ cells and antibody production.

30. Rankin AL, Guay H, Herber D, et al. IL-21 receptor is required for the systemic accumulation of activated $\mathrm{B}$ and $\mathrm{T}$ lymphocytes in MRL/MpJ-Fas(lpr/lpr)/J mice. J Immunol. 2012;188(4):1656-67.

31. De Nitto D, Sarra M, Pallone F, et al. Interleukin-21 triggers effector cell responses in the gut. World J Gastroenterol. 2010;16 (29):3638-41.

32. van Heel DA, Franke L, Hunt KA, et al. A genome-wide association study for celiac disease identifies risk variants in the region harboring IL2 and IL21. Nat Genet. 2007;39:827-9.

33. Asano K, Ikegami H, Fujisawa T, et al. Molecular scanning of interleukin-21 gene and genetic susceptibility to type 1 diabetes. Hum Immunol. 2007;68(5):384-91.

34. Teixeira VH, Pierlot $\mathrm{C}$, Migliorini $\mathrm{P}$, et al. Testing for the association of the KIAA1109/Tenr/IL2/IL21 gene region with rheumatoid arthritis in a European family-based study. Arthritis Res Ther. 2009;11:2-6.

35. Costanzo A, Chimenti MS, Botti E, et al. IL-21 in the pathogenesis and treatment of skin diseases. J Dermatol Sci. 2010;60(2):61-6.

36. Botti E, Spallone G, Caruso R, et al. Psoriasis, from pathogenesis to therapeutic strategies: IL-21 as a novel potential therapeutic target. Curr Pharm Biotechnol. 2012;13(10):1861-7.

37. - Caruso R, Botti E, Sarra M, et al. Involvement of interleukin-21 in the epidermal hyperplasia of psoriasis. Nat Med. 2009;15:10135. This is one of the first studies to describe the role of IL-21 in psoriasis and a therapeutic efficacy in humanized mouse model.

38. Sarra M, Caruso R, Cupi ML, et al. IL-21 promotes skin recruitment of CD4(+) cells and drives IFN- $\gamma$-dependent epidermal hyperplasia. J Immunol. 2011;186(9):5435-42.

39. Sugiyama H, Gyulai R, Toichi E, et al. Dysfunctional blood and target tissue $\mathrm{CD} 4+\mathrm{CD} 25$ high regulatory $\mathrm{T}$ cells in psoriasis: mechanism underlying unrestrained pathogenic effector $\mathrm{T}$ cell proliferation. J Immunol. 2005;174:164-73. 
40. Di Cesare A, Di Meglio P, Nestle FO. The IL-23/Th17 axis in the immunopathogenesis of psoriasis. J Investig Dermatol. 2009; 129:1339-50.

41. Wong CK, Wong PTY, Tam LS, et al. Elevated production of B cell chemokine CXCL13 is correlated with systemic lupus erythematosus disease activity. J Clin Immunol. 2010;30(1):45-52.

42. Dolff S, Abdulahad WH, Westra J, et al. Increase in IL-21 producing T-cells in patients with systemic lupus erythematosus. Arthritis Res Ther. 2011;13(5):157.

43. Sawalha AH, Kaufman KM, Kelly JA, et al. Genetic association of interleukin-21 polymorphisms with systemic lupus erythematosus. Ann Rheum Dis. 2008;67(4):458-61.

44. Webb R, Merrill JT, Kelly JA, et al. A polymorphism within IL21R confers risk for systemic lupus erythematosus. Arthritis Rheum. 2009;60(8):2402-7.

45. Simpson N, Gatenby PA, Wilson A, et al. Expansion of circulating $T$ cells resembling follicular helper $T$ cells is a fixed phenotype that identifies a subset of severe systemic lupus erythematosus. Arthritis Rheum. 2010;62(1):234-44.

46. Crispin JC, Martínez A, Alcocer-Varela J. Quantification of regulatory $\mathrm{T}$ cells in patients with systemic lupus erythematosus. J Autoimmun. 2003;21(3):273-6.

47. Valencia X, Yarboro C, Illei G, et al. Deficient CD4+CD25 high $\mathrm{T}$ regulatory cell function in patients with active systemic lupus erythematosus. J Immunol. 2007;178(4):2579-88.

48. - Herber D, Brown TP, Liang S, et al. IL-21 has a pathogenic role in a lupus-prone mouse model and its blockade with IL-21R.Fc reduces disease progression. J Immunol. 2007;178:3822-30. This is one of the first studies to describe a therapeutic efficacy in SLE mouse model.

49. Odegard JM, Marks BR, DiPlacido LD, et al. ICOS-dependent extrafollicular helper $\mathrm{T}$ cells elicit IgG production via IL-21 in systemic autoimmunity. J Exp Med. 2008;205(12):2873-86.

50. Izui S, Ibnou-Zekri N, Fossati-Jimack L, et al. Lessons from BXSB and related mouse models. Int Rev Immunol. 2000;19:447-72.

51. Bubier JA, Sproule TJ, Foreman O, et al. A critical role for IL-21 receptor signaling in the pathogenesis of systemic lupus erythematosus in BXSB-Yaa mice. Proc Natl Acad Sci U S A. 2009;106:1518-23.

52. • Bubier JA, Bennett SM, Sproule TJ, et al. Treatment of BXSB-Yaa mice with IL-21R-Fc fusion protein minimally attenuates systemic lupus erythematosus. Ann N Y Acad Sci. 2007;1110:590-601. This is one of the first studies to describe a therapeutic efficacy in SLE mouse model.

53. Sarra M, Monteleone G. Interleukin-21: a new mediator of inflammation in systemic lupus erythematosus. J Biomed Biotechnol. 2010;201:294582.

54. Kabesch M, Halonen M, Holberg CJ, et al. A cluster of seven tightly linked polymorphisms in the IL-13 gene is associated with total serum IgE levels in three populations of white children. J Allergy Clin Immunol. 2000;105:506-13.

55. Kabesch M, Schedel M, Carr D, et al. IL-4/IL-13 pathway genetics strongly influence serum IgE levels and childhood asthma. J Allergy Clin Immunol. 2006;117:269-74.

56. Sicherer SH, Leung DY. Advances in allergic skin disease, anaphylaxis, and hypersensitivity reactions to foods, drugs, and insects. J Allergy Clin Immunol. 2007;119(6):1462-9.
57. Larché M, Akdis CA, Valenta R. Immunological mechanisms of allergen-specific immunotherapy. Nat Rev Immunol. 2006;6 (10):761-71.

58. - Jin H, Oyoshi MK, Le Y, et al. IL-21R is essential for epicutaneous sensitization and allergic skin inflammation in humans and mice. J Clin Invest. 2009;119:47-60. This is one of the first studies to describe a therapeutic efficacy in AD mouse model.

59. Homey B, Steinhoff M, Ruzicka T, et al. Cytokines and chemokines orchestrate atopic skin inflammation. J Allergy Clin Immunol. 2006;118:178-89.

60. Spergel JM, Mizoguchi E, Brewer JP, et al. Epicutaneous sensitization with protein antigen induces localized allergic dermatitis and hyperresponsiveness to methacholine after single exposure to aerosolized antigen in mice. J Clin Invest. 1998;101:1614-22.

61. Lin SC, Chuang YH, Yang YH, et al. Decrease in interleukin-21 in children suffering with severe atopic dermatitis. Pediatr Allergy Immunol. 2011;22(8):869-75.

62. Hennino A, Berard F, Guillot I, et al. Pathophysiology of urticaria. Clin Rev Allergy Immunol. 2006;30:3-11.

63. Huilan Z, Runxiang L, Bihua L, et al. Role of the subgroups of T, $\mathrm{B}$, natural killer lymphocyte and serum levels of interleukin-15, interleukin-21 and immunoglobulin $\mathrm{E}$ in the pathogenesis of urticaria. J Dermatol. 2010;37(5):441-7.

64. Shang XZ, Ma KY, Radewonuk J, et al. IgE isotype switch and IgE production are enhanced in IL-21-deficient but not IFN-gammadeficient mice in a Th2-biased response. Cell Immunol. 2006;241:66-74.

65. Herrmann M, Voll RE, Kalden JR. Etiopathogenesis of systemic lupus erythematosus. Immunol Today. 2000;21(9):424-6.

66. Distler JH, Jüngel A, Kowal-Bielecka $\mathrm{O}$, et al. Expression of interleukin-21 receptor in epidermis from patients with systemic sclerosis. Arthritis Rheum. 2005;52(3):856-64.

67. Kawaguchi, Yasushi, Takagi, et al. Augmentation of IL-21 receptor by endothelin-1 via endothelin receptor B in skin fibroblasts from systemic sclerosis [abstract]. Arthritis Rheum. 2009;60(Suppl 10):1808. Presented at the 2009 ACR/ARHP Annual Scientific Meeting, Philadelphia, PA, October 16-21, 2009.

68. Kurzinski K, Torok KS. Cytokine profiles in localized scleroderma and relationship to clinical features. Cytokine. 2011;55(2):157-64.

69. Fossiez F, Djossou O, Chomarat P, et al. T-cell interleukin-17 induces stromal cells to produce proinflammatory and hematopoietic cytokines. J Exp Med. 1996;183(6):2593-603.

70. McGonagle D, McDermott MF. A proposed classification of the immunological diseases. PLoS Med. 2006;3:297.

71. • Pineton de Chambrun M, Wechsler B, Geri G, et al. New insights into the pathogenesis of Behcet's disease. Autoimmun Rev. 2012;11(10):687-98. A review that give a very good overview of the role of IL-21 in Behçet's disease.

72. Chi W, Zhu X, Yang P, et al. Upregulated IL-23 and IL-17 in Behcet patients with active uveitis. Investig Ophthalmol Vis Sci. 2008;49:3058-64.

73. Chi W, Zhou S, Yang P, et al. CD4+ T cells from Behçet patients produce high levels of IL-17. Yan Ke Xue Bao. 2011;26:6-13.

74. Geri G, Terrier B, Rosenzwajg M, et al. Critical role of IL-21 in modulating $\mathrm{T}(\mathrm{H}) 17$ and regulatory $\mathrm{T}$ cells in Behcet disease. $\mathrm{J}$ Allergy Clin Immunol. 2011;128:655-64. 